Case Report

\title{
Posterior Reversible Encephalopathy Syndrome with Stroke in Puerperal Woman with High Titer of Anti-Phospholipid IgM Antibody
}

\author{
Hiroto Hirashima, ${ }^{1}$ Masahiro Iwamoto ${ }^{D},{ }^{2}$ Tadashi Ozawa, ${ }^{3}$ Shigeyoshi Kijima, \\ Shigeki Matsubara $₫{ }^{1}{ }^{1}$ and Akihide Ohkuchi $\oplus^{1}$ \\ ${ }^{1}$ Department of Obstetrics and Gynecology, Jichi Medical University School of Medicine, 3311-1 Yakushiji, Shimotsuke-shi, \\ Tochigi 329-0498, Japan \\ ${ }^{2}$ Division of Rheumatology and Clinical Immunology, Department of Medicine, Jichi Medical University School of Medicine, \\ 3311-1 Yakushiji, Shimotsuke-shi, Tochigi 329-0498, Japan \\ ${ }^{3}$ Division of Neurology, Department of Internal Medicine, Jichi Medical University School of Medicine, 3311-1 Yakushiji, \\ Shimotsuke-shi, Tochigi 329-0498, Japan \\ ${ }^{4}$ Department of Radiology, Jichi Medical University School of Medicine, 3311-1 Yakushiji, Shimotsuke-shi, Tochigi 329-0498, Japan
}

Correspondence should be addressed to Akihide Ohkuchi; okuchi@jichi.ac.jp

Received 29 June 2018; Accepted 21 November 2018; Published 3 December 2018

Academic Editor: Mehmet A. Osmanağaoğlu

Copyright (c) 2018 Hiroto Hirashima et al. This is an open access article distributed under the Creative Commons Attribution License, which permits unrestricted use, distribution, and reproduction in any medium, provided the original work is properly cited.

Posterior reversible encephalopathy syndrome with stroke is very rare in puerperal women. A 36-year-old nulliparous woman with both rheumatoid arthritis and recurrent pregnancy loss, probably due to a high titer of anti-phospholipid IgM antibody, was referred at 10 weeks of gestation. Low-dose aspirin at $100 \mathrm{mg} /$ day and heparin calcium subcutaneous injection at 10,000 units/day were started before pregnancy and stopped at $35^{+6}$ and $40^{+2}$ weeks, respectively. She transabdominally delivered a male infant weighing $3,344 \mathrm{~g}$ at $40^{+5}$ weeks. A tonic-clonic seizure abruptly occurred without either hypertension or proteinuria 5 days after delivery. Intracerebral hemorrhage involving an area of $2 \mathrm{~cm}$ in diameter in the right frontal lobe and subarachnoid hemorrhage with PRES were confirmed. Seizure recurred 2 days after the initial episode. She showed severe headache and mild disturbance of consciousness but no neurological findings. We suggested that a high titer of anti-phospholipid IgM antibody might be associated with stroke.

\section{Introduction}

As described in the first report of posterior reversible encephalopathy syndrome (PRES) by Hinchey et al. [1], PRES is a condition that is associated with not only preeclampsia and eclampsia, which are very common in cases of PRES in pregnancy or the puerperal period, but also numerous systemic conditions in nonobstetric patients of all ages. However, PRES with intracranial hemorrhage (ICH), that is, stroke, in a pregnant woman with neither preeclampsia nor eclampsia is very rare. In addition, PRES in patients with either rheumatoid arthritis (RA) or a high titer of antiphospholipid antibody is markedly rare; to the best of our knowledge, only 3 cases of PRES in patients with RA and
4 cases of PRES in patients positive for anti-phospholipid antibody have been reported [2-8].

Here, we present a woman with both RA and recurrent pregnancy loss probably due to a high titer of antiphospholipid IgM antibody, but without hypertension, who suffered PRES with stroke (intracerebral and subarachnoid hemorrhage), accompanied by two tonic-clonic seizures, occurring at 5 and 7 days after delivery.

\section{Case Report}

A 36-year-old nulliparous woman with past histories of both RA and recurrent pregnancy loss (repeated abortion), probably due to a high titer of anti-phospholipid IgM 


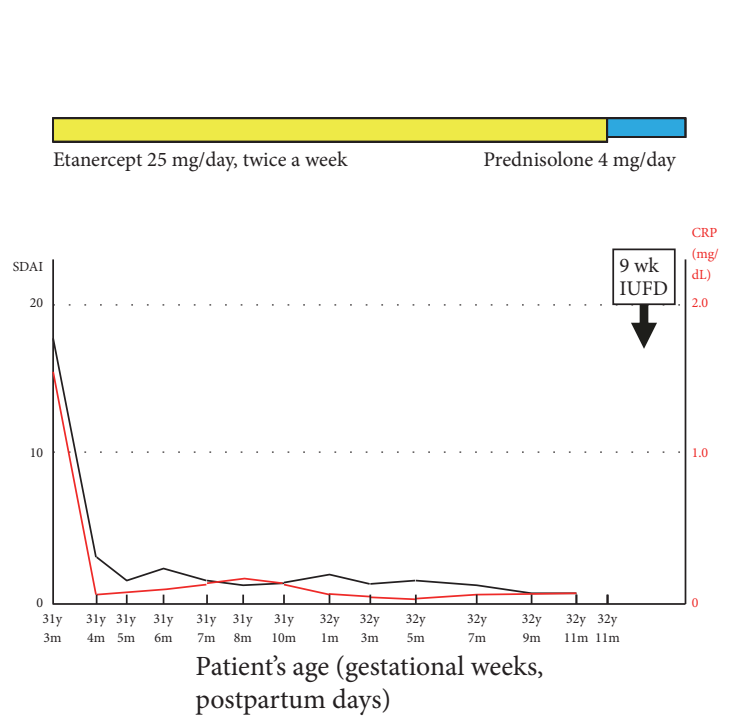

(a)
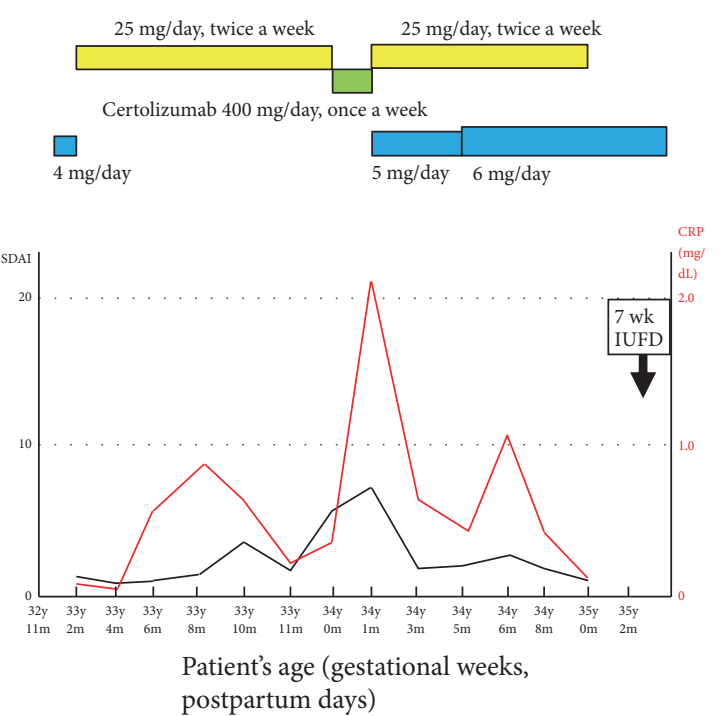

(b)

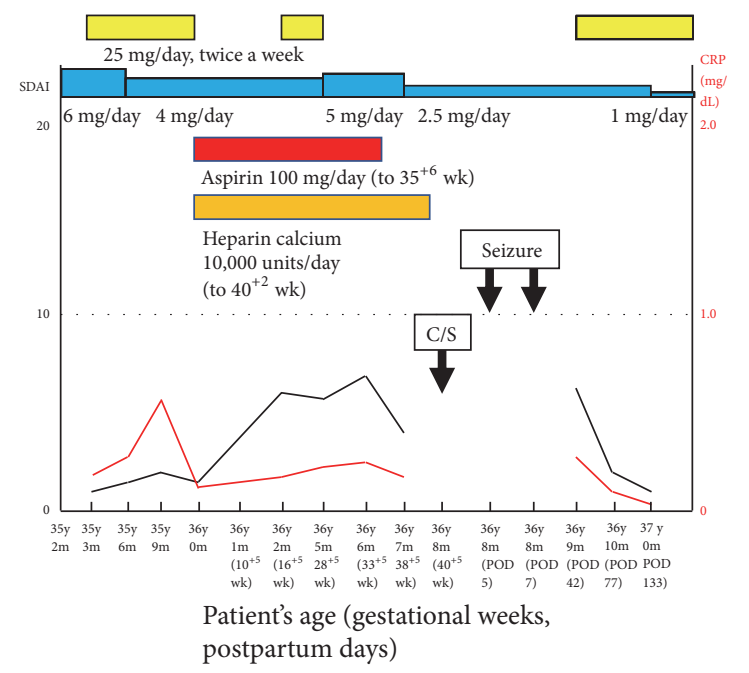

(c)

FIGURE 1: The levels of the simplified disease activity index (SDAI), which was the sum of the number of tender joints, the number of swollen joints, patient global assessment of disease activity by visual analogue scale (VAS), care provider global assessment of disease activity by VAS, and the levels of C-reactive protein (CRP) $(\mathrm{mg} / \mathrm{dL})$, and serum C-reactive protein (CRP) from the onset of rheumatoid arthritis (RA) at 31 years and 3 months old to 133 days after the current delivery. (a) The levels of SDAI and CRP from 31 years and 3 months old to 32 years and 11 months old when she became pregnant for the first time, which resulted in intrauterine fetal death (IUFD) at 9 weeks of gestation. (b) The levels of SDAI and CRP from 32 years and 11 months old to 35 years and 2 months old when she became pregnant for the second time, which also resulted in IUFD at 7 weeks of gestation. (c) The levels of SDAI and CRP from 35 years and 2 months old to postpartum day 133 in the delivery. The used drugs are shown at the top of the charts. wk, weeks of gestation; y, years; m, months; C/S, cesarean section; POD, postoperative days.

antibody, was referred to our tertiary center at 10 weeks of gestation.

RA had been diagnosed at 31 years old, and etanercept at $25 \mathrm{mg} /$ day, twice a week, was started. Within 1 month, the simplified disease activity index (SDAI) had improved from 18.1 to 2.6 (Figure 1(a)). SDAI was the sum of the number of tender joints, the number of swollen joints, patient global assessment of disease activity using a visual analogue scale (VAS), care provider global assessment of disease activity by VAS, and the level of C-reactive protein (CRP)
(mg/dL). At 32 years old, she became pregnant, and etanercept was changed to prednisolone at $4 \mathrm{mg}$ /day; however, intrauterine fetal death (IUFD) occurred at 9 weeks. Thereafter, prednisolone was changed to etanercept at $25 \mathrm{mg} /$ day (Figure 1(b)). Since she desired to become pregnant at 35 years old, etanercept was discontinued before pregnancy; she soon became pregnant, but at 7 weeks, IUFD occurred again. Thereafter, etanercept at $25 \mathrm{mg} /$ day, twice a week, was restarted with a decreased dose of prednisolone at $4 \mathrm{mg} /$ day (Figure 1(c)). 


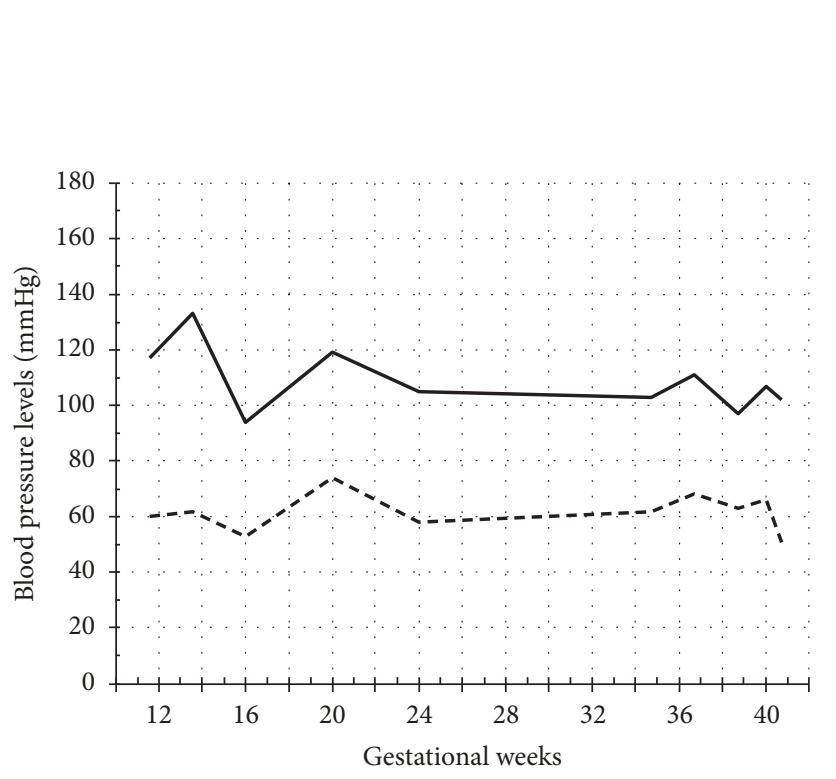

(a)

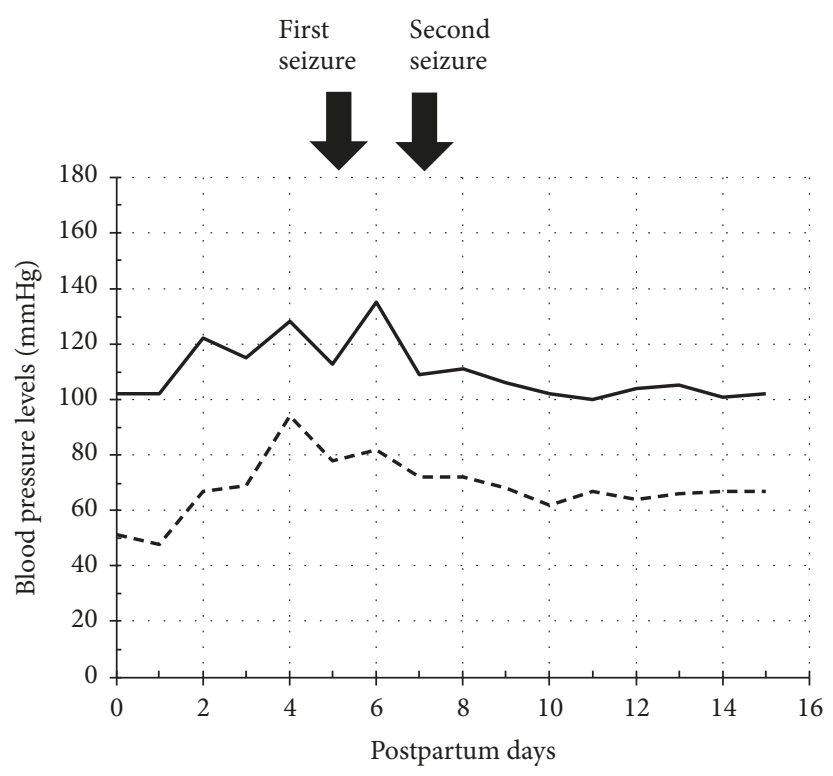

(b)

FIGURE 2: Systolic blood pressure (SBP) and diastolic blood pressure (DBP) during pregnancy and the puerperal period. (a) SBP and DBP during pregnancy. Solid jagged lines indicate SBP, whereas broken jagged lines indicate DBP. (b) SBP and DBP from delivery to 16 days after delivery. Seizure occurred 5 and 7 days after delivery.

Because of repeated abortion and the discovery of a high titer of anti-cardiolipin IgM antibody (twice $\geq 80 \mathrm{U} / \mathrm{mL}$ with intervals of $\geq 3$ months [reference range: $<8 \mathrm{U} / \mathrm{mL}]$ ), lowdose aspirin at $100 \mathrm{mg} /$ day and heparin calcium subcutaneous injection at 10,000 units/day were started before pregnancy, with the discontinuation of etanercept. At 36 years old, she became pregnant for a third time. Because SDAI was increased to 6.8 at $16^{+5}$ weeks, etanercept at $25 \mathrm{mg}$ /day, twice a week, was reinitiated. However, etanercept was discontinued at $28^{+5}$ weeks, because the British Society of Rheumatology (BSR) and British Health Professionals in Rheumatology (BHPR) guidelines on prescribing drugs in pregnancy and breastfeeding recommended the usage of etanercept until the end of the second trimester [9]. Then, prednisolone was increased from 4 to $5 \mathrm{mg} /$ day from $28^{+5}$ weeks; however, prednisolone was decreased from 5 to $2.5 \mathrm{mg} /$ day from $38^{+5}$ weeks, because the SDAI was decreased from 7.9 to 4.9. Taken together, the SDAI scores during pregnancy were slightly higher than those before the current pregnancy.

Aspirin was stopped at $35^{+6}$ weeks, and heparin was stopped at $40^{+2}$ weeks. She transabdominally delivered a male infant weighing $3,344 \mathrm{~g}$ at $40^{+5}$ weeks due to arrest of labor following its induction. A tonic-clonic seizure abruptly occurred without either hypertension or proteinuria 5 days after delivery; her blood pressure at the first seizure was $113 / 78 \mathrm{mmHg}$, and that at the second seizure was $109 / 72 \mathrm{mmHg}$ (Figure 2). Severe headache preceded the convulsion and continued after the seizure for almost 7 days. After the seizure, her consciousness was mildly disturbed (Japan Coma Scale I-3). There were no visual changes, and no hemiplegia. Computed tomography (CT) disclosed intraparenchymal hemorrhage with $2 \mathrm{~cm}$ diameter in the right frontal lobe (Figure 3(a)) and a fluid-attenuated inversion recovery (FLARE) image obtained by magnetic resonance imaging (MRI) disclosed subarachnoid hemorrhage at the right Sylvian fissure (Figure 3(b)). MRI also revealed PRES in the bilateral frontal, temporal, and posterior lobes (Figures 3(c)-3(f)). Laboratory data revealed that she was not complicated by either thrombocytopenia or disseminated intravascular coagulation. Levetiracetam at $1,000 \mathrm{mg} /$ day, carbazochrome sodium sulfonate hydrate at $100 \mathrm{mg} / \mathrm{day}$, and tranexamic acid at $1,000 \mathrm{mg} /$ day were prescribed. Seizure recurred 2 days after the first one; however, cerebral images on CT did not change compared with those 2 days before the seizure (Figure 3(g)). MRI findings were ameliorated 1 month after the seizure (Figures $3(\mathrm{~h})-3(\mathrm{k})$ ).

We did not formally evaluate SDAI around the time of seizure occurrence; however, in retrospect, the patient reported that the patient global assessment of disease activity by VAS around the seizures was milder than in the term pregnancy period. Etanercept at $25 \mathrm{mg}$ /day, twice a week, was restarted 42 days after delivery; and prednisolone was changed from 2.5 to $1.0 \mathrm{mg} /$ day 133 days after delivery (Figure 1(c)). SDAI 3 months after delivery had markedly improved to 0.88 .

\section{Discussion}

The PRES patient with stroke was complicated by RA. In retrospect, the patient reported that the patient global assessment of disease activity by VAS around the seizures was milder than in the term pregnancy period; thus, we suggested that activity of RA may have not been associated with the PRES and stroke. However, to the best of our knowledge, only 3 cases of RA with PRES have been reported [2-4]. These 4 cases including our case may only be the tip of the iceberg, 


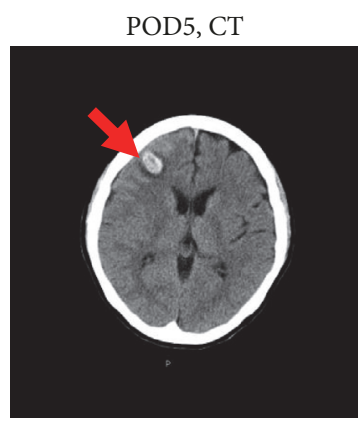

(a)

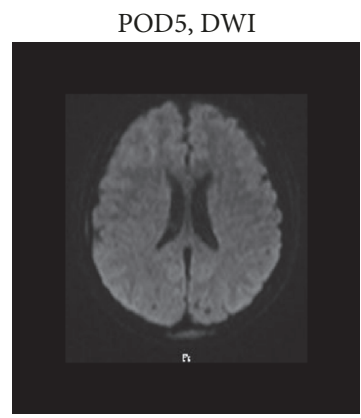

(e)

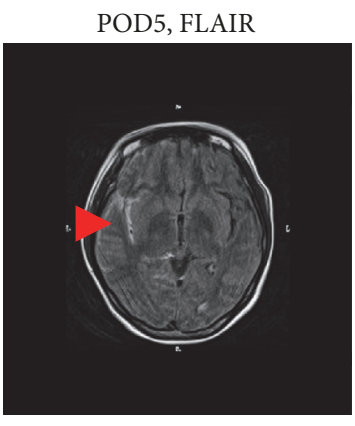

(b)

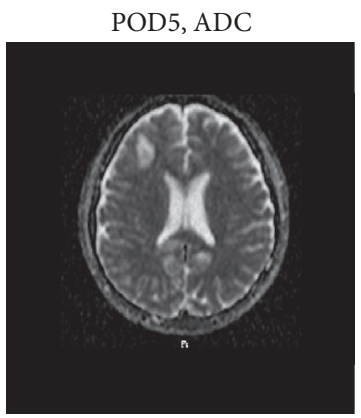

(f)

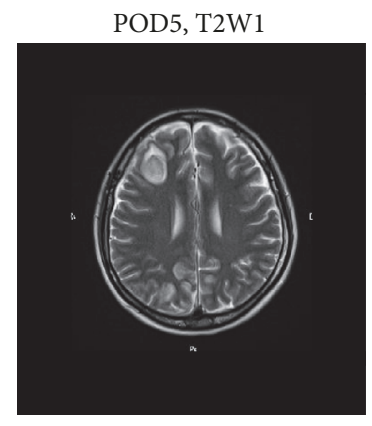

(c)

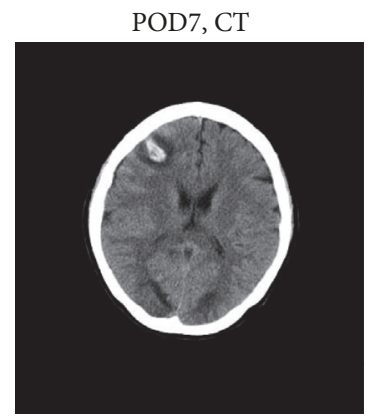

(g)

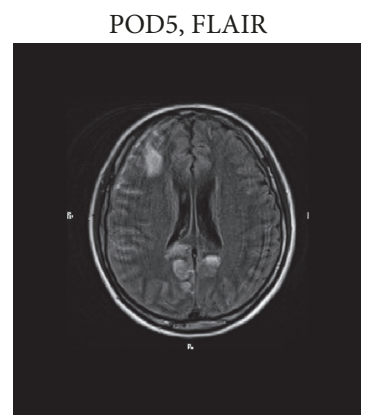

(d)

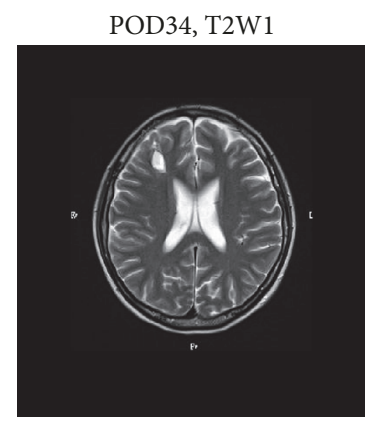

(h)

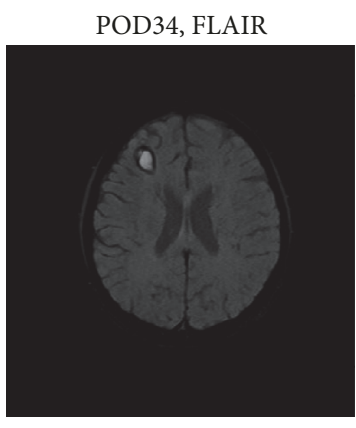

(i)

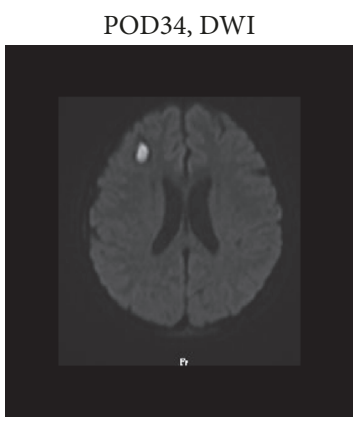

(j)

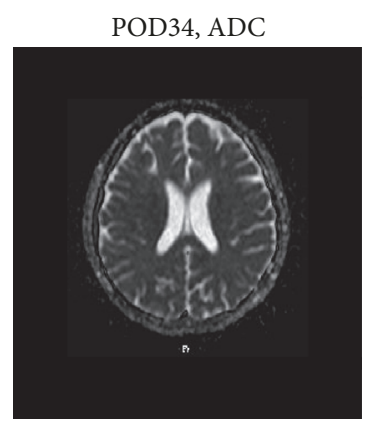

(k)

FIgURE 3: (a) CT image 5 days after delivery, just after the occurrence of tonic-clonic seizure. Arrow indicates intraparenchymal hemorrhage with $2 \mathrm{~cm}$ diameter in the right frontal lobe. (b) FLAIR image on MRI 5 days after delivery, just after the occurrence of tonic-clonic seizure. Arrowhead indicates subarachnoid hemorrhage at the right Sylvian fissure. (c) T2-weight image on MRI 5 days after delivery, just after the first seizure. (d) FLAIR image on MRI 5 days after delivery. (e) DWI on MRI 5 days after delivery. (f) ADC mapping on MRI 5 days after delivery. (g) CT image 7 days after delivery, just after the second tonic-clonic seizure. (h) T2-weighted image on MRI 34 days after delivery. (i) FLAIR image on MRI 34 days after delivery. (j) DWI on MRI 34 days after delivery. (k) ADC mapping on MRI 34 days after delivery. CT, computed tomography; MRI, magnetic resonance imaging; POD, postoperative days; T2W1, T2-weighted; FLAIR, fluid-attenuated inversion recovery; DWI, diffusion-weighted image; ADC, apparent diffusion coefficient.

because autoimmune disease is a well-known risk factor for the occurrence of PRES.

A high titer of anti-phospholipid IgM antibody may have been associated with PRES, although, to the best of our knowledge, only 4 cases of PRES have been reported in patients with a high titer of anti-phospholipid antibody [5-8]. Systemic lupus erythematosus (SLE) is a wellknown disease that is a risk factor for the occurrence of PRES $[10,11]$, and SLE is sometimes accompanied by antiphospholipid antibodies [12]. We speculate that the antiphospholipid antibody may be related to endothelial injury and the inflammation of endothelial cells, thus, leading to PRES.

This case was very rare, showing PRES with stroke 5 days after delivery, without complication by either preeclampsia or HELLP syndrome. A high titer of anti-phospholipid antibody might have been associated with the occurrence of stroke, because the frequency of being positive for anti-phospholipid antibody in young patients with stroke has been estimated as $17.2 \%$ [13], whereas the frequency of being positive for antiphospholipid antibody in healthy adults was around 3\% [14]. These data suggest that pregnant women with a high titer of 
anti-phospholipid antibody might have a high risk of stroke during pregnancy and the puerperal period. In our patient, LDA and heparin were discontinued during the puerperal period.

In conclusion, a puerperal woman complicated with both RA and recurrent pregnancy loss, probably due to a high titer of anti-phospholipid IgM antibody, showed PRES with stroke 5 days after delivery, although she was not complicated with either hypertension or proteinuria. We speculate that a high titer of anti-phospholipid IgM antibody may have been associated with the occurrence of stroke. Since case reports of PRES with either RA or a high titer of anti-phospholipid antibody are still limited [2-8], the accumulation of such cases is necessary to elucidate whether RA or a high titer of anti-phospholipid antibody is a risk factor for the occurrence of PRES. In addition, from a clinical point of view, puerperal woman with a high titer of anti-phospholipid antibody may have a high risk of stroke. Therefore, the accumulation and evaluation of patients with stroke during pregnancy and the puerperal period is necessary to elucidate the role of high titer of anti-phospholipid antibody in the occurrence of cerebral vascular disease.

\section{Conflicts of Interest}

The authors declare that they have no conflicts of interest.

\section{References}

[1] J. Hinchey, C. Chaves, B. Appignani et al., "A reversible posterior leukoencephalopathy syndrome," The New England Journal of Medicine, vol. 334, no. 8, pp. 494-500, 1996.

[2] C. Hart, M. O. Kinney, and M. O. McCarron, "Posterior reversible encephalopathy syndrome and oral methotrexate," Clinical Neurology and Neurosurgery, vol. 114, no. 6, pp. 725-727, 2012.

[3] J. H. Zimering and A. Mesfin, "Posterior reversible encephalopathy syndrome following elevated mean arterial pressures for cervical spinal cord injury," The Journal of Spinal Cord Medicine, vol. 41, no. 1, pp. 111-114, 2018.

[4] T. Makino, I. Kamitsukasa, and S. Ito, "Reversible cerebral vasoconstriction syndrome due to atovaquone," Case Reports in Neurology, vol. 9, pp. 304-308, 2017.

[5] D. Renard, A. Dutray, A. Le Quellec, and D. Milhaud, "Reversible posterior leukoencephalopathy syndrome in catastrophic antiphospholipid syndrome," Cerebrovascular Disease, vol. 24, no. 1, pp. 141-143, 2007.

[6] A. Lateef and A. Y. Lim, "Case reports of transient loss of vision and systemic lupus erythematosus," ANNALS Academy of Medicine Singapore, vol. 36, pp. 146-149, 2007.

[7] M. Bandettini di Poggio, G. Murdaca, F. Puppo, and A. Primavera, "Antiphospholipid syndrome and reversible posterior leukoencephalophaty syndrome," Seminars in Arthritis and Rheumatism, vol. 40, no. 3, pp. e9-e10, 2010.

[8] K. Larmour, G. Lewis, G. Benson, and J. Hanko, “The challenges of antiphospholipid syndrome: experience from diagnosis to self-care," BMJ Case Reports, vol. 2014, 2014.

[9] J. Flint, S. Panchal, A. Hurrell et al., "BSR and BHPR guideline on prescribing drugs in pregnancy and breastfeeding-Part I: Standard and biologic disease modifying anti-rheumatic drugs and corticosteroids," Rheumatology, vol. 55, no. 9, pp. 1693-1697, 2016.
[10] A. Mak, B. P. L. Chan, I. B. Yeh et al., "Neuropsychiatric lupus and reversible posterior leucoencephalopathy syndrome: A challenging clinical dilemma," Rheumatology, vol. 47, no. 3, pp. 256-262, 2008.

[11] A. Dhillon, C. Velazquez, and C. Siva, "Rheumatologic diseases and posterior reversible encephalopathy syndrome: two case reports and review of the literature," Rheumatology International, vol. 32, no. 12, pp. 3707-3713, 2012.

[12] U. İlgen, M. E. Yayla, A. Ateş et al., "Antiphospholipid antibodies and non-thrombotic manifestations of systemic lupus erythematosus," Lupus, vol. 27, no. 4, pp. 665-669, 2018.

[13] S. Sciascia, G. Sanna, M. A. Khamashta et al., "The estimated frequency of Antiphospholipid antibodies in young adults with cerebrovascular events: A systematic review," Annals of the Rheumatic Diseases, vol. 74, no. 11, pp. 2028-2033, 2015.

[14] C. B. Chighizola, L. Andreoli, G. R. De Jesus, A. Banzato, G. J. Pons-Estel, and D. Erkan, "The association between antiphospholipid antibodies and pregnancy morbidity, stroke, myocardial infarction, and deep vein thrombosis: A critical review of the literature," Lupus, vol. 24, no. 9, pp. 980-984, 2015. 


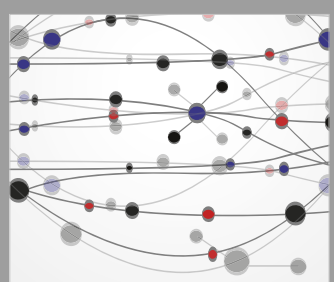

The Scientific World Journal
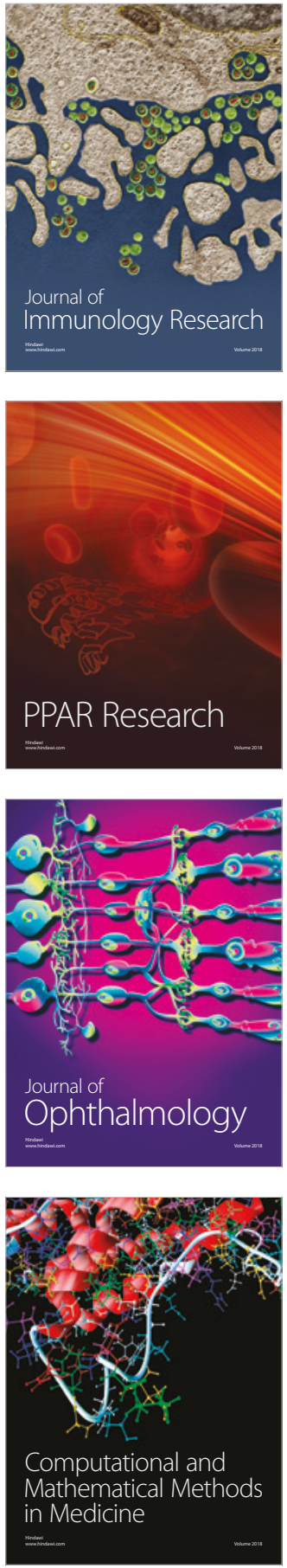

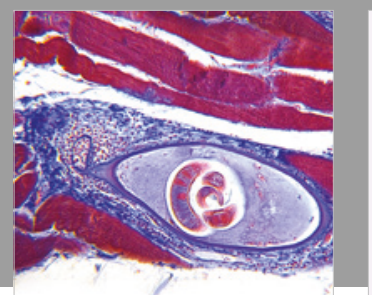

Gastroenterology Research and Practice

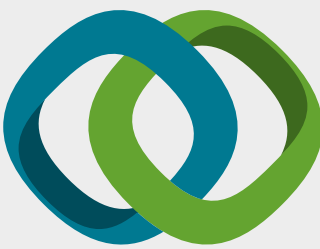

\section{Hindawi}

Submit your manuscripts at

www.hindawi.com
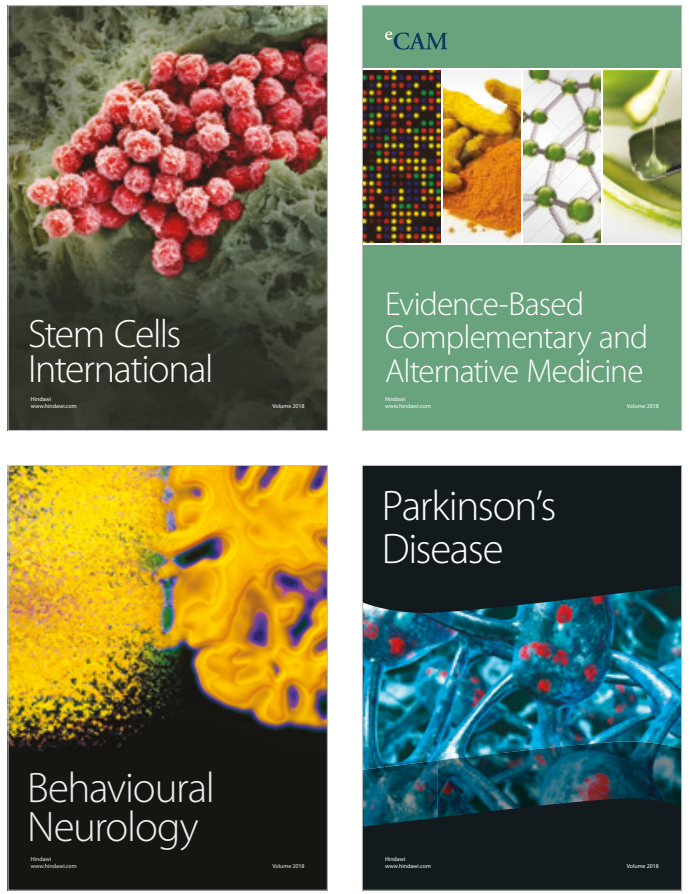

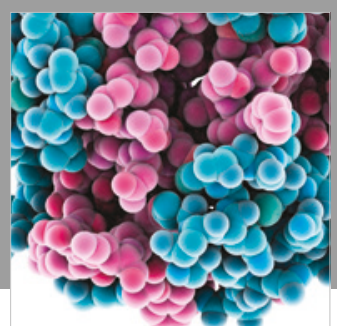

ournal of

Diabetes Research

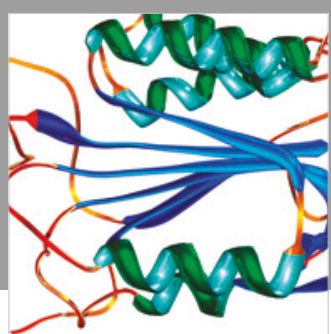

Disease Markers
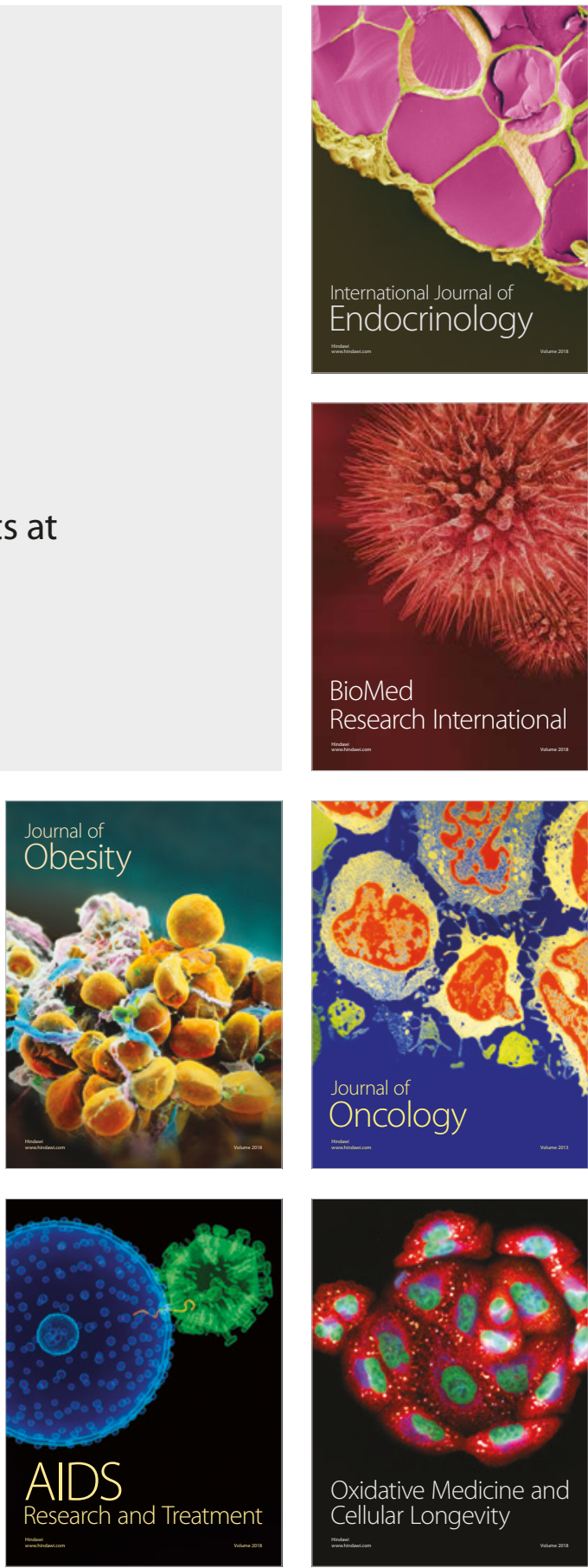